\title{
EFEITOS DE DEJETO DE SUÍNOS NA FORMA LÍQUIDA E DE COMPOSTO ORGÂNICO NA ATIVIDADE DO METRIBUZIN ${ }^{1,2}$
}

\author{
SILVÉRIO DE P. FREITAS ${ }^{3}$, TOCIO SEDIYAMA ${ }^{4}$, ANTÔNIO A. DA SILVA ${ }^{4}$, FRANCISCO A. FERREIRA ${ }^{4}$ CARLOS S. \\ SEDIYAMA ${ }^{4}$
}

\section{RESUMO}

Este trabalho objetivou avaliar a atividade do metribuzin em função da adição de doses de dejeto de suínos na forma líquida e de composto orgânico. Foram conduzidos dez experimentos utilizando delineamento experimental inteiramente casualizado, com quatro repetições, em condições de casa-de-vegetação do Departamento de Fitotecnia da UFV. Foram avaliados cinco substratos: areia lavada, solo Podzólico VermelhoAmarelo argilo arenoso, da região de Ponte NovaMG misturado com doses correspondentes a 0,20 , 40 e $60 \mathrm{~m}^{3} \mathrm{ha}^{-1}$ de dejeto líquido e $0,30,60$ e $90 \mathrm{t}$ $\mathrm{ha}^{-1}$ de composto orgânico associados a 10 doses de metribuzin. A aplicação do metribuzin foi feita com pulverizador costal pressurizado a $\mathrm{CO}_{2}$, após a semeadura de cinco sementes/vaso da planta indicadora (Cucumis sativus L.) híbrido caipira AG.221. A irrigação dos vasos foi realizada, três vezes ao dia, por meio de simulador de chuvas, mantendo-se a umidade dos substratos em aproximadamente $80 \%$ da capacidade de campo. Aos 14 dias após a aplicação dos tratamentos foi realizada a colheita dos experimentos, seguida pela determinação da biomassa fresca da parte aérea da planta indicadora. As concentrações dos herbicidas, que resultaram em $50 \%$ de inibição do crescimento da planta teste $\left(\mathrm{I}_{50}\right)$, foram calculadas a partir de equações ajustadas por meio de análise de regressão. A adsorção do metribuzin aumentou linearmente com adição de dejeto líquido ou composto orgânico ao solo, sendo que a maior adsorção ocorreu com a adição do composto orgânico. Para a maior dose de dejeto líquido $\left(60 \mathrm{~m}^{3} \mathrm{ha}^{-1}\right)$, a relação de adsorção foi 4,79 e o aumento na adsorção em relação ao solo foi $28,01 \%$. Entretanto, com a adição da maior dose do composto orgânico (90 t ha ${ }^{-1}$ ), a relação de adsorção foi 6,63 e o aumento na adsorção $32,51 \%$.

Palavras chave: Adsorção, herbicida, resíduos, planta indicadora.

\section{ABSTRACT \\ Metribuzin soil bioactivity as a function of swine liquid manure and organic matter}

The objective of this work was to evaluate metribuzin bioactivity as a function of adding swine liquid manure doses and organic matter produced from crushed sugar-cane. It was conducted ten experiments using entirely randomized experimental design with four replicates in greenhouse conditions at the Departamento de Fitotecnia da Universidade
Federal de Viçosa/MG. Five substrates were evaluated: washed sand, Yellow-Red clayishsandy Podzolic soil, from Ponte Nova - Minas Gerais State, mixed with 0, 20, 40 and $60 \mathrm{~m}^{3} \mathrm{ha}^{-1}$ doses of swine liquid manure and 0, 3060 and $90 \mathrm{t} \mathrm{ha}^{-1}$ doses of organic matter associated to ten doses of metribuzin. This herbicide was applied using a $\mathrm{CO}_{2}$ pressurized costal sprayer after

\footnotetext{
${ }^{1}$ Recebido para publicação em 25/11/97 e na forma revisada em 11/02/99

${ }^{2}$ Parte da tese do primeiro autor apresentada à UFV para obtenção do título de Doutor em Fitotecnia.

${ }_{3}^{3}$ Professor de Manejo de Plantas Daninhas do LFIT/CCTA/UENF. Av. Alberto Lamego, 2000, CEP 28015-620, Campos/RJ. Tel:024 7263752.E - mail silverio@uenf.br.

${ }^{4}$ Professores do Departamento de Fi totecnia/UFV. CEP 36.570-000, Viçosa/MG.
} 
sowing five seeds of the indicator plant (Cucumis sativus L.) AG. 221 caipira hybrid in each vase. The vases were irrigated three times a day using a rainfall simulator and the substrate moisture was maintained about $80 \%$ of field capacity. Fourteen days after applying treatments, the haversting of experiments was realized, followed by determination of fresh biomass of the indicatorplant aerial part. The herbicide concentrations resulting in 50\% inhibition of the test-plant growth $\left(\mathrm{I}_{50}\right)$ were calculated from equations adjusted by regression analysis. The adsorption relations and adsorption increasing were calculated as a function of the addition of swine

\section{INTRODUÇÃO}

Os dejetos de suínos são 100 vezes mais poluentes que o esgoto urbano. Uma granja de suínos com 2.000 animais, polui o equivalente a uma cidade com 36.000 habitantes (Konzen, 1980). Com o propósito de solucionar este problema, tem-se observado, em trabalhos recentes, resultados promissores quanto à utilização racional desses dejetos em forma líquida incorporada ao solo ou em forma de composto orgânico, com respostas positivas para produtividade das culturas e aspecto visual do produto colhido (Scherer \& Baldissera, 1995, Matos et al., 1995 e Freitas et al., 1995).

A matéria orgânica é um componente do solo de extrema importância no processo de bioatividade dos herbicidas devido sua elevada capacidade de troca catiônica e superfície específica, além da presença de grupos funcionais carboxílicos, fenólicos, alcoólicos e amínicos. Ela pode estar associada à fração argila, formando os complexos argilo-orgânicos, que podem variar em quantidade e qualidade. Solos com a mesma porcentagem de matéria orgânica podem apresentar diferenças marcantes em relação a suas propriedades físico-químicas, influenciando de modo diferente a atividade de um mesmo herbicida. Isto acontece principalmente porque a origem e o grau de decomposição da matéria orgânica podem ser diferentes (Obrigawitch et al., manure and organic matter doses. Metribuzin adsorption increased linearly with the addition of swine liquid manure and organic matter to soil. The greater adsorption occurred with the addition of organic matter. Also, it was observed that for the highest dose of swine liquid manure $\left(60 \mathrm{~m}^{3}\right.$ $\mathrm{ha}^{-1}$ ), the adsorption relation was 4.79 , and in relation to soil without organic matter the adsorption increasing was $28.01 \%$. However whit the addition of the organic matter highest dose $\left(90 \mathrm{t} \mathrm{ha}^{-1}\right)$, the adsorption relation was 6.63 and the increase in adsorption was $32.51 \%$.

Key words: Adsorption, substrates, herbicide, indicator plant.

1981; Peter \& Weber, 1985, Walker, 1987, Sousa, 1994 e Oliveira, 1995).

Qualquer herbicida aplicado ao solo fica não disponível em algum nível, tendo sua atividade reduzida, proporcionalmente à quantidade adsorvida, permanecendo em estado passivo, não disponível para os processos biológicos, físicos e químicos até à ocorrência do processo de adsorção negativa. $\mathrm{O}$ herbicida deve permanecer disponível no solo até o momento em que sua finalidade seja cumprida, qual seja, manter a cultura livre de plantas daninhas até o momento, em que aquelas que germinarem, não mais irão interferir na produtividade da cultura ou prejudicar a colheita e/ou a qualidade do produto. A persistência longa do herbicida no solo (maior que o ciclo da cultura) é altamente indesejável pois pode intoxicar culturas sucessivas, as quais podem ser sensíveis a estes resíduos (Blanco, 1979 e Silva, 1985).

Em estudos sobre adsorção de herbicidas, podem ser utilizados métodos químicos ou bioensaios com organismos vivos (Klingman \& Ashtom, 1975). Todavia, diversos fatores podem influenciar a resposta do bioensaio aos herbicidas tais como o tipo de solo, teores de matéria orgânica, de água e de nutrientes, pH do solo, localização da semente no solo, densidade de semeadura, luz, temperatura, cultivar e interação entre herbicidas (Appleby, 1985 e Onyeniy \& Akinyemiju, 1990). 
O método mais comum para realização de bioensaios consiste na realização de ensaios preliminares procurando-se identificar a espécie altamente sensível (planta-teste) ao produto a ser avaliado. Após esta etapa, diferentes doses do produto devem ser aplicados sobre esta planta, ou no substrato onde esta espécie foi semeada, procurando-se determinar a dose capaz de inibir em $50 \%$ o desenvolvimento de um processo, ou uma função fisiológica, ou ainda, o crescimento da planta teste, elaborando-se uma curva padrão de respostas (Aldus, 1976).

Este trabalho objetivou avaliar a atividade do metribuzin em função da adição de doses de dejeto de suínos na forma líquida e de composto orgânico.

\section{MATERIAL E MÉTODOS}

Foram conduzidos, em casa-de-vegetação, no campus da Universidade Federal de Viçosa, dez experimentos, inteiramente casualizados, com dez tratamentos (Tabela 1) e quatro repetições para cada substrato, sendo que cada vaso foi considerado como uma parcela experimental. Foram utilizados areia lavada e material de solo Podzólico Vermelho-Amarelo Câmbico, fase terraço, coletado na Fazenda Experimental da EPAMIG em Ponte Nova-MG e misturado com diferentes concentrações de dejeto de suínos, na forma líquida, e de composto orgânico produzido a partir de bagaço de cana-de-açúcar e dejeto de suínos.

Tabela 1. Doses de metribuzin aplicadas nos diferentes substratos. Viçosa-MG, 1995.

\begin{tabular}{|c|c|c|c|c|c|c|c|c|c|c|}
\hline Substratos & & & & & $\overline{\mathrm{de} M}$ & ouzin & $\left.\mathrm{ha}^{-1}\right)$ & & & \\
\hline Areia lavada ${ }^{\underline{1}}$ & 0 & 2 & 4 & 6 & 8 & 10 & 12 & 14 & 16 & 18 \\
\hline Solo+0 $\mathrm{m}^{3}$ ha $^{-1}$ dejeto ${ }^{1 /}$ & 0 & 12 & 24 & 36 & 48 & 60 & 72 & 84 & 96 & 108 \\
\hline Solo+20 $\mathrm{m}^{3} \mathrm{ha}^{-1}$ dejeto $^{1 /}$ & 0 & 16 & 32 & 48 & 64 & 80 & 96 & 112 & 128 & 144 \\
\hline Solo+40 $\mathrm{m}^{3} \mathrm{ha}^{-1}$ dejeto ${ }^{1 /}$ & 0 & 16 & 32 & 48 & 64 & 80 & 96 & 112 & 128 & 144 \\
\hline Solo+60 $\mathrm{m}^{3} \mathrm{ha}^{-1}$ dejeto $^{1 /}$ & 0 & 16 & 32 & 48 & 64 & 80 & 96 & 112 & 128 & 144 \\
\hline Areia lavada ${ }^{2 /}$ & 0 & 2 & 4 & 6 & 8 & 10 & 12 & 14 & 16 & 18 \\
\hline Solo +0 t ha $^{-1}$ composto $^{2 /}$ & 0 & 12 & 24 & 36 & 48 & 60 & 72 & 84 & 96 & 108 \\
\hline Solo +30 t ha $^{-1}$ composto ${ }^{2}$ & 0 & 16 & 32 & 48 & 64 & 80 & 96 & 112 & 128 & 144 \\
\hline Solo+60 t ha $^{-1}$ composto & 0 & 16 & 32 & 48 & 64 & 80 & 96 & 112 & 128 & 144 \\
\hline Solo+90 t ha ${ }^{-1}$ composto $^{\underline{2}}$ & 0 & 16 & 32 & 48 & 64 & 80 & 96 & 112 & 128 & 144 \\
\hline
\end{tabular}

${ }^{1 /}$ Ensaio realizado em agosto de 1995 .

${ }^{2} /$ Ensaio realizado em março de 1995 .

Os substratos foram coletados após a mistura de material orgânico com o solo, realizada em condições de campo, por ocasião do preparo de leiras para o plantio de batata-doce. Sendo que em uma área, foram adicionadas doses correspondentes a $0,20,40$ e $60 \mathrm{~m}^{3} \mathrm{ha}^{-1}$ de dejeto de suínos na forma líquida e na outra, 0, 30, 60 e $90 \mathrm{tha}^{-1}$ de composto orgânico.
Inicialmente, foram avaliados os efeitos de dez doses de metribuzin (Tabela 1) sobre a biomassa fresca da planta de pepino (Cucumis sativus) cultivada em areia lavada, para elaboração da equação padrão de adsorção. Essa areia foi passada em peneira de malha de dois milímetros e, em seguida, tratada, durante 36 horas com ácido clorídrico (solução comercial) diluído em água na proporção de $600 \mathrm{ml}$ de ácido para cada dez litros 
de água. Após este período, a areia foi lavada com água corrente até retirar o excesso de ácido, ficando com o pH próximo a 6,5 em água desmineralizada.

Além da areia lavada, os demais substratos utilizados nos ensaios foram também passados em peneira de malha de quatro milímetros e secados ao ar até atingirem 2,5\% de umidade residual. Em seguida, foram colocados em vasos plásticos com capacidade para $430 \mathrm{~cm}^{3}$ e inteiramente recobertos com sacos de polietileno. A mesma massa de substratos foi colocada em cada vaso.

A semeadura da planta teste (pepino) foi realizada a uma profundidade de $1,5 \mathrm{~cm}$, colocando-se cinco sementes por vaso. $\mathrm{O}$ metribuzin foi aplicado à superfície do solo seco nas doses correspondentes a cada tratamento (Tabela 1), utilizando um pulverizador costal pressurizado com $\mathrm{CO}_{2}$, provido de uma barra de meio metro, contendo dois bicos tipo leque "Teejet" 80.03, gastando o equivalente a 200 litros de calda por ha.

A primeira irrigação foi realizada logo após aplicação do metribuzin, utilizando-se um simulador de chuva para vasos, previamente construído para este fim. Inicialmente, nos vasos contendo a areia, aplicou-se um volume de água correspondente a uma chuva de $9 \mathrm{~mm}$, com duração de seis minutos, enquanto nos substratos contendo solo, este volume correspondeu a uma chuva de $12 \mathrm{~mm}$ durante oito minutos. Quinze horas após esta primeira irrigação, o volume de água em cada substrato foi aumentado até atingir $80 \%$ da capacidade de campo, previamente determinada para cada substrato. Para manter o teor de água constante nos substratos, foram feitas irrigações três vezes ao dia, visando repor a água evapotranspirada.

Aos 14 dias após aplicação dos tratamentos, foi realizada a colheita dos experimentos. A parte aérea da planta foi colhida, e depois sua biomassa fresca foi determinada.

$\mathrm{Na}$ interpretação dos resultados, utilizouse a análise de regressão, e a partir de equações ajustadas, calculou-se o $\mathrm{I}_{50}$ correspondente a cada substrato.
Com base nos valores de $\mathrm{I}_{50}$, determinouse a Razão de Adsorção (RA), que permite comparar a capacidade adsortiva para diferentes substratos, que, segundo Sousa (1994), é calculada pela equação:

$$
R A=\frac{I_{50} \text { substrato }-I_{50} \text { areia }}{I_{50} \text { areia }}
$$

O Aad (aumento da adsorção) permite verificar o aumento percentual da capacidade adsortiva, em função da adição ao solo de dejeto ou composto orgânico, foi obtido pela equação :

$$
\operatorname{Aad}(\%)=\frac{\mathrm{I}_{50}(\text { solo }+\mathrm{X}) \times 100}{\mathrm{I}_{50} \text { solo }},
$$

onde $\mathrm{X}$ é igual a dejeto de suíno ou composto orgânico.

Com base nos valores de $\mathrm{I}_{50}$, utilizando a análise de regressão, foram determinados os modelos matemáticos que melhor explicassem a adsorção do herbicida, em função de doses de dejeto de suínos na forma líquida ou de composto orgânico adicionados ao solo.

\section{RESULTADOS E DISCUSSÃO}

O modelo linear foi o que melhor se ajustou para explicar a variação de biomassa em plantas de pepino, em função de doses de metribuzin aplicados nos diferentes substratos (Figuras 1, 2, 3 e 4).

A dose de metribuzin calculada a partir da equação ajustada, capaz de inibir, em $50 \%$ a biomassa da parte aérea das plantas de pepino $\left(\mathrm{I}_{50}\right)$ cultivadas em areia lavada foi de $9,16 \mathrm{~g} \mathrm{ha}^{-1}$ para os ensaios realizados em março de $1995 \mathrm{em}$ um período com alta luminosidade e alta temperatura (Figura 1). Todavia, para os ensaios conduzidos durante o mês de agosto (ocorrência de dias nublados e baixas temperaturas) observou-se que $o$ valor de $\mathrm{I}_{50}$ foi $10,69 \mathrm{~g} \mathrm{ha}^{-1}$, concordando com os resultados obtidos por Appleby (1985) e Onyeniy \& Akinyemiju (1990), segundo os quais fatores climáticos entre outros, influenciam a resposta dos 
bioensaios aos herbicidas, razão pela qual foram feitas duas equações-padrão.

Considerando a adsorção do metribuzin no solo sem adição de dejeto de suínos ou de composto orgânico, nas duas épocas em que os experimentos foram conduzidos verifica-se resposta linear na redução da biomassa da planta teste em função do aumento de doses do herbicida resultando valores de $\mathrm{I}_{50}$ correspondentes a $48,37 \mathrm{e}$ $52,75 \mathrm{~g} / \mathrm{ha}$ de metribuzin, para os plantios da planta teste em março e agosto, respectivamente (Figura 2).

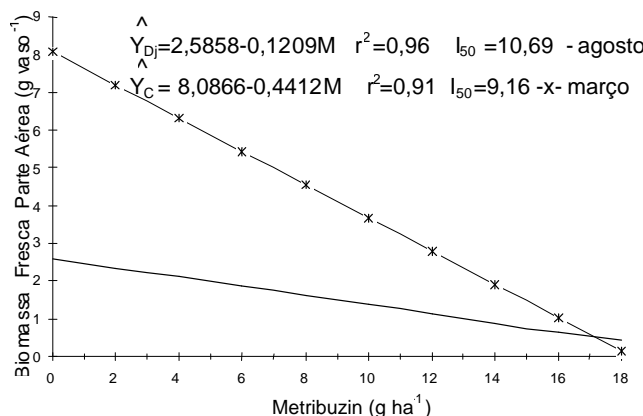

Figura 1. Resposta do crescimento, em biomassa verde, da parte aérea das plantas de pepino cultivadas em areia lavada, tratada com diferentes doses de metribuzin. Viçosa-MG, 1995.

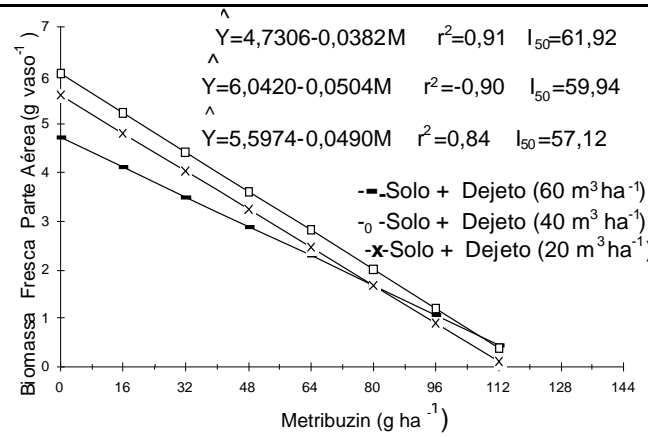

Figura 3. Resposta do crescimento, em biomassa verde, da parte aérea das plantas de pepino cultivadas em solo com 20, 40 e $60 \mathrm{~m}^{3} \mathrm{ha}^{-1}$ de dejeto de suínos, tratados com diferentes doses de metribuzin. Viçosa-MG, 1995.

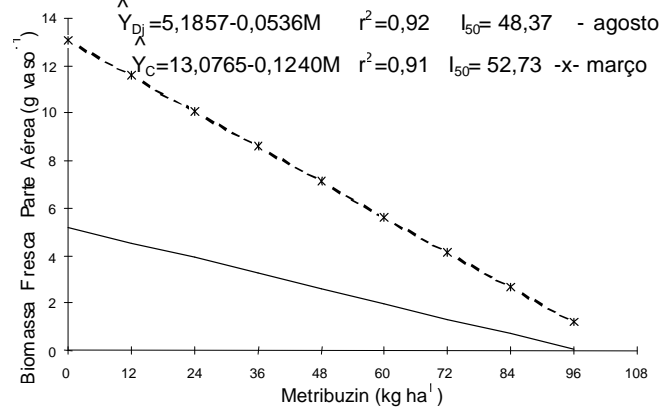

Figura 2. Resposta do crescimento, em biomassa verde, da parte aérea das plantas de pepino cultivadas em solo, tratado com diferentes doses de metribuzin. Viçosa-MG, 1995.

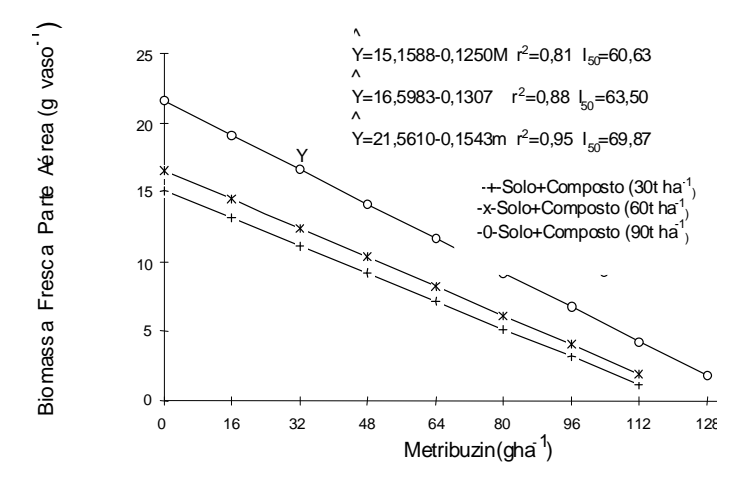

Figura 4.Resposta do crescimento, em biomassa verde, da parte aérea das plantas de pepino cultivadas em solo com 30,60 e 90 t ha $^{-1}$ de composto orgânico, tratados com diferentes doses de metribuzin. Viçosa-MG, 1995.
Observa-se que com a adição do dejeto de suínos na forma líquida ao solo nas doses 20,40 e $60 \mathrm{~m}^{3} \mathrm{ha}^{-1}$, os $\mathrm{I}_{50}$ foram 57,$12 ; 59,94$ e 61,92 , respectivamente (Figura 3). Todavia, ao adicionar 30, 60 e $90 \mathrm{t} \mathrm{ha}^{-1}$ de composto orgânico ao solo, os valores de $I_{50}$ foram: 60,63; 63,50 e 69,87, respectivamente (Figura 4).

A relação de adsorção aumentou com o incremento das doses, tanto para dejeto de suínos na forma líquida adicionado ao solo quanto na de composto orgânico, verificando-se relação de 
adsorção correspondentes a 3,52; 4,34; 4,61 e 4,79 para o solo com adição de $0,20,40$ e $60 \mathrm{~m}^{3} \mathrm{ha}^{-1} \mathrm{de}$ dejeto de suínos forma líquida, respectivamente e 4,$76 ; 5,62 ; 5,93$ e 6,63 para o solo com adição de $0,30,60$ e $90 \mathrm{t} \mathrm{ha}^{-1}$ na forma de composto orgânico, respectivamente (Tabela 2). Estes resultados concordam com os trabalhos de Peter \& Weber (1985) e Oliveira (1995), os quais observaram maior adsorção do metribuzin em função da adição de matéria orgânica ao solo.

Tabela 2. Doses de metribuzin que inibiram em $50 \%$ o crescimento da planta teste $\left(\mathrm{I}_{50}\right)$, relação de adsorção e aumento percentual da adsorção do metribuzin em função da adição ao solo de dejeto de suínos na forma líquida e de composto orgânico. Viçosa-MG, 1995.

\begin{tabular}{|c|c|c|c|}
\hline Substratos & $\begin{array}{c}\mathrm{I}_{50} \\
\left(\mathrm{~g} \mathrm{ha}{ }^{-1}\right) \\
\end{array}$ & Relação de Adsorção & $\begin{array}{c}\text { Aumento de Adsorção } \\
(\%)\end{array}$ \\
\hline Areia lavada ${ }^{1 /}$ & 10,69 & - & - \\
\hline Solo $+0 \mathrm{~m}^{3}$ ha $^{-1}$ dejeto ${ }^{1 /}$ & 48,37 & 3,52 & - \\
\hline Solo $+20 \mathrm{~m}^{3}$ ha $^{-1}$ dejeto ${ }^{1 /}$ & 57,12 & 4,34 & 18,09 \\
\hline Solo $+40 \mathrm{~m}^{3}$ ha $^{-1}$ dejeto ${ }^{1 /}$ & 59,94 & 4,61 & 23,92 \\
\hline Solo+60 $\mathrm{m}^{3}$ ha $^{-1}$ dejeto ${ }^{\underline{1 /}}$ & 61,92 & 4,79 & 28,01 \\
\hline Areia lavada $\stackrel{2}{\stackrel{2}{ }}$ & 9,16 & - & \\
\hline Solo+0 $\mathrm{t} \mathrm{ha}^{-1}$ composto $^{2 /}$ & 52,73 & 4,76 & - \\
\hline Solo +30 t ha $^{-1}$ composto ${ }^{2 /}$ & 60,63 & 5,62 & 14,98 \\
\hline Solo+60 t ha $^{-1}$ composto ${ }^{2}$ & 63,50 & 5,93 & 20,42 \\
\hline Solo +90 t ha $^{-1}$ composto $\stackrel{2}{~}$ & 69,87 & 6,63 & 32,51 \\
\hline
\end{tabular}

${ }^{1 /}$ Ensaio realizado em agosto de 1995 .

${ }^{2 /}$ Ensaio realizado em março de 1995 .

Na Figura 5, comparando-se a adsorção do metribuzin no solo sem adição de dejeto de suínos com a adsorção no solo com adição de 20,40 e 60 $\mathrm{m}^{3} \mathrm{ha}^{-1}$ observou-se que os aumentos percentuais da adsorção foram 18,$09 ; 23,92$ e 28,01 , respectivamente. Todavia, ao comparar a adsorção do metribuzin no solo sem adição de composto orgânico com a adsorção no solo com adição de 30, 60 e 90 tha $^{-1}$ de composto orgânico, verificouse que os aumentos percentuais da adsorção foram 14,98; 20,42 e 32,51, respectivamente (Figura 6).

Estes resultados estão de acordo com aqueles obtidos por Schimittd (1973), Savage (1976), Silva \& Silva (1991), Peter \& Weber
(1985), Veline (1992) e Oliveira (1995), que verificaram que a adsorção do metribuzin está diretamente correlacionada com o teor de argila e principalmente com o teor de matéria orgânica devido às modificações que esta causa nas características químicas e físicas do solo.

Quando se analisou os valores de $\mathrm{I}_{50}$ para os diferentes substratos (Figuras 7 e 8) verificouse aumento linear em função de doses de dejetos de suínos e de composto orgânico. Resultados semelhantes foram obtidos por Silva (1975), que observou aumento linear na adsorção do metribuzin, quando o teor de matéria orgânica adicionada à areia lavada variava entre 0 a $8 \%$. 


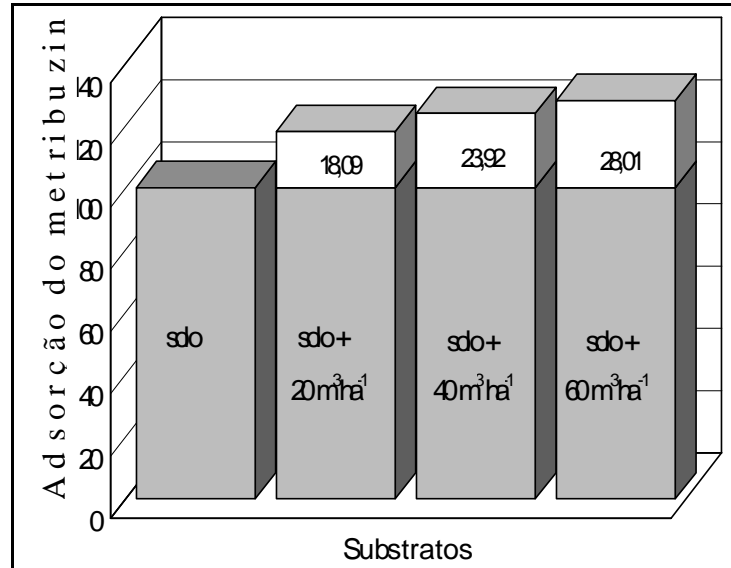

Figura 5. Aumento da adsorção do metribuzin (\%) em solo com adição $0,20,40$ e $60 \mathrm{~m}^{3}$ ha $^{-1}$ de dejeto de suínos na forma líquida. Viçosa-MG, 1995

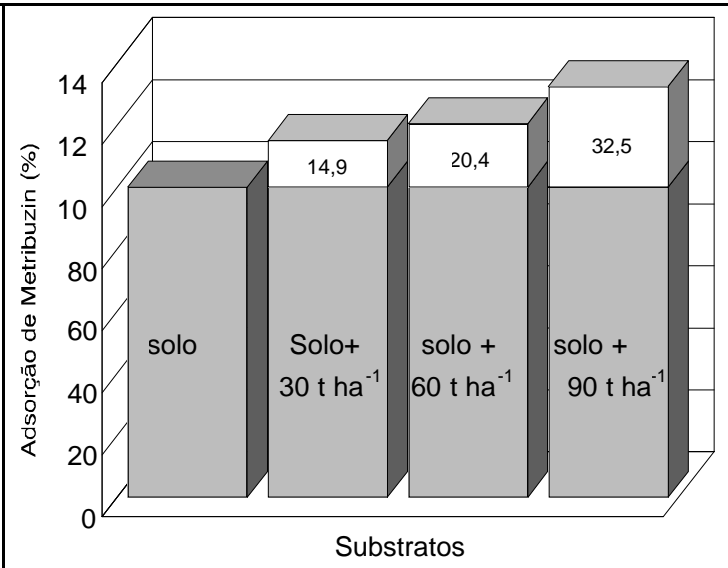

Figura 6. Aumento da adsorção do metribuzin (\%) em solo com adição de $0,30,60$ e $90 \mathrm{t}$ ha $^{-1}$ de composto orgânico. Viçosa-MG, 1995.

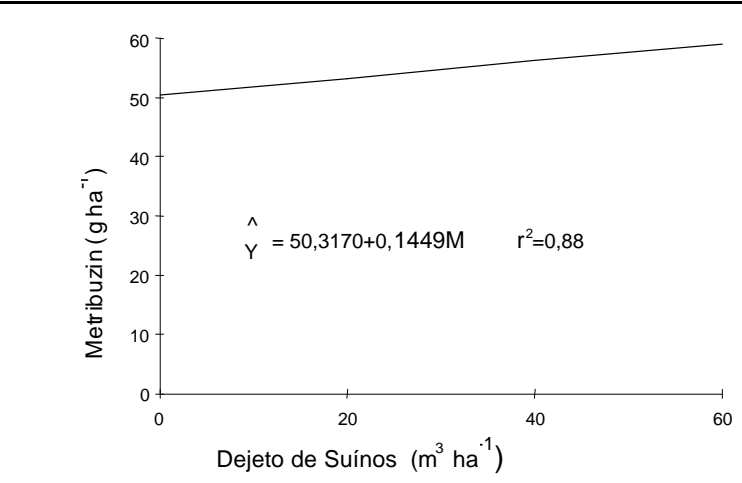

Figura 7. Doses de metribuzin capazes de inibir $50 \%$ do crescimento da planta de pepino $\left(\mathrm{I}_{50}\right)$, em função de doses de dejeto de suínos. Viçosa-MG, 1995.

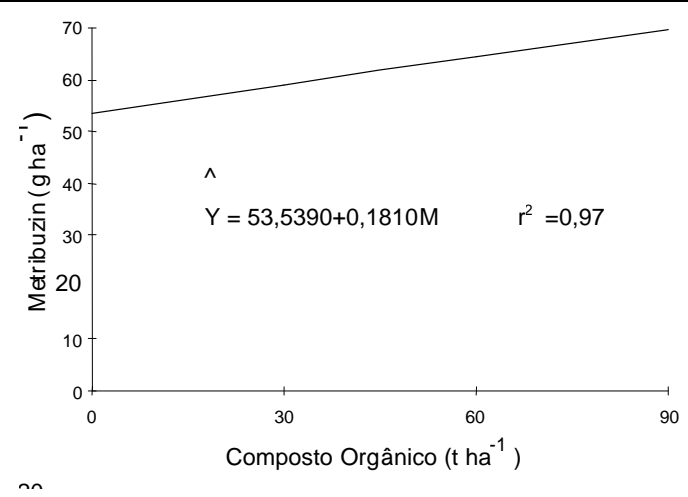

Figura 8. Doses de metribuzin capazes de inibir $50 \%$ do crescimento da planta de pepino $\left(\mathrm{I}_{50}\right)$, em função de doses de composto orgânico. Viçosa-MG, 1995.

\section{LITERATURA CITADA}

ALDUS, L.J. Physiology, biochemistry and ecology. 2.ed. London: Academic. Press, 1976. 546p.

APPLEBY, A.P. Factors in examining fate of herbicides in soil with biossays. Weed Sci., v.33, p.22-26, 1985.
BLANCO, H.G. Destino, comportamento e resíduos dos herbicidas no solo. O Biológico, v.45, p.225-248, 1979.

FREITAS, S.P. de; SEDIYAMA T.; SEDIYAMA M.A. N.; VIDIGAL S.M.; SILVA, A.da. Resposta de algumas hortaliças à adubação com compostos orgânicos e dejeto líquido de suínos. I SEMINÁRIO MINEIRO SOBRE MANEJO E UTILIZAÇÃO DE DEJETOS DE 
SUÍNOS. Anais, 1995 Ponte Nova-MG. EPAMIG, Ed. Viçosa, p.55-67.

KLIGMAN, G.C., ASHTOM, F.M. Principles and pratices: weed science .New York: John Wiley, 1975. 431p.

KONZEN, E.A. Avaliação quantitativa e qualitativa dos dejetos de suínos em crescimento e terminação, manejados em forma líquida. Belo Horizonte: UFMG, Escola de Veterinária, 1980. 56p. Dissertação ( Mestrado em Veterinária) Universidade Federal de Minas Gerais, Escola de Veterinária, 1980.

MATOS, A.T. de, SEDIYAMA, M.A.N., VIDIGAL, S. M., et al. Propriedades químicas e microbiológicas do solo influenciadas pela aplicação de dejeto líquido de suíno. In: ENCONTRO DA SEÇÃO BRASILEIRA DA INTERNATIONAL ASSOCATION FOR IMPACT ASSESMENT (IAIA), 1995, Belo Horizonte. Anais... Belo Horizonte: IAIA/UFMG, 1995. p.403-411.

OBRIGAWITCH, T., HOWNS, F.M., ABERNARTHY, J.R., et al. Adsorption, desorption and mobility of metolachor. Soil Weed Sci., v.29, p.332-6, 1981.

OLIVEIRA, M.F. de. Adsorção, lixiviação e persistência de Flumioxazin e Metribuzin em diferentes solos. ViçosaMG: UFV, 1995. 71p. Dissertação (Mestrado em Fitotecnia) - Universidade Federal de Viçosa, 1995.

ONYENIY, A., AKINYEMIJU, O.A. Use of biossays of herbicide persistence studies in the humid tropics. Turrialba, v.40, p.26571, 1990.

PETER, C.J., WEBER, J.B. Adsorption, mobility and efficacy of metribuzin as influenced by soil properties, Weed Sci., v.33, p.86873, 1985.

SAVAGE, K.E. Herbicidal activity and selectivity of metribuzin. Soil Weed Scien., v.24, p.525-528, 1976.

SCHERER, E.E., BALDISSERA, I.T., ROSSO, A. de. Utilização de dejetos suínos como fertilizantes. Aspectos práticos do manejo de dejetos de suínos. Florianópolis: EPAGRE/EMBRAPA, CNPSA, 1995. 106p.

SCHIMITD, R.R. Calculations on the herbicidal activity of metribuzin in relation to various soils propieties.In...SYMPOSIUM HERBICIDES, SOIL, 1973. Proceedings... [S.1.]: European Weed Researche Council, 1973. p.24-40.

SILVA, J.F.da. Herbicidal activity and selectivity of metribuzin. Lafaette: Purdue University, 1975. 58p. Tese (P.h.D. Thesis) - Purdue University, 1975.

SILVA, J.F. da, SILVA, J.F. da. Formulações misturas e seletividade de herbicidas In: CURSO DE ESPECIALIZAÇÃO POR TUTORIA À DISTÂNCIA. MÓDULO: PLANTAS DANINHAS E SEU CONTROLE. Herbicidas. Brasília - D.F.: ABEAS, 1991. 75p. (Apostila)

SILVA, J.F. da, SILVA, J.F. da, SILVA, R.F. da, CONDÉ, A.R. Lixiviação e inativação do Metribuzin em dois tipos de solos . Planta Daninha, v.4, p.78-82, 1985.

SOUSA, A.P. de. Atividade de oxyfluorfen, 2,4$D$ e glyphosate, em solos de diferentes texturas na presença e na ausência de composto orgânico. Viçosa, MG: UFV., 1994. 71 p. Dissertação (Mestrado em Solos e Nutrição de Plantas) Universidade Federal de Viçosa, 1994. 
VELINI, E.D. Comportamento de herbicidas no solo. In: SIMPÓSIO SOBRE MANEJO INTEGRADO DE PLANTAS DANINHAS EM HORTALIÇAS, 1992, Botucatu. Anais... Botucatu: UNESP,
1992. p. 44-64.

WALKER, A. Herbicide persistence in soil. Weed Science Society of America, 1987. In: Separata de: Weed Sci., v.3, p.1-17, 1987. 